\title{
COMMON FIXED POINTS FOR GENERALIZED ASYMPTOTICALLY NONEXPANSIVE MAPPINGS
}

\author{
SUMIT CHANDOK AND T. D. NARANG
}

\begin{abstract}
A common fixed point theorem for noncommuting generalized asymptotically nonexpansive mappings has been obtained in convex metric spaces. As an application, a result on the set of best approximation is also derived for such class of mappings. The proved results unify and extend some of the known results on the subject.
\end{abstract}

\section{Introduction}

Common fixed points of two commuting mappings satisfying some contractive or nonexpansive type conditions have been studied by many researchers (see [1]-[4],[7],[9]-[12] and references cited therein). The introduction of noncommuting mappings such as weakly commuting, $R$-weakly commuting, $R$-subweakly commuting, compatible, weakly compatible and $C_{q}$-commuting mappings, was a turning point in the fixed point arena. A wider class of nonexpansive mapings, known as asymptotically nonexpansive mappings, was introduced by Goebel and Kirk [5]. Vijayaraju and Hemavathy [12] proved some common fixed point theorems and approximation results by extending the results of Beg et al. [2] to generalized asymptotically $S$-nonexpansive and $C_{q}$-commuting mappings in normed linear spaces. This paper deals with the study of common fixed point theorem for generalized asymptotically $S$ nonexpansive and noncommuting mappings in convex metric spaces. As an application, we also establish a result on the set of best approximation. The results proved in the paper unify and extend some known results in the literature.

\section{Definitions and Preliminaries}

For a metric space $(X, d)$, a continuous mapping $W: X \times X \times[0,1] \rightarrow X$ is said to be a convex structure on $X$ if for all $x, y \in X$ and $\lambda \in[0,1]$,

$$
d(u, W(x, y, \lambda)) \leq \lambda d(u, x)+(1-\lambda) d(u, y)
$$

Corresponding author: Sumit Chandok.

2010 Mathematics Subject Classification. 41A50, 47H10, 54H25.

Key words and phrases. $C_{q}$-commuting, convex metric space, starshaped set and generalized asymptotically nonexpansive mappings.

The research has been supported by the U.G.C. India under the Emeritus Fellowship. 
holds for all $u \in X$. The metric space $(X, d)$ together with a convex structure is called a convex metric space [13].

A subset $K$ of a convex metric space $(X, d)$ is said to be a convex set [13] if $W(x, y, \lambda) \in K$ for all $x, y \in K$ and $\lambda \in[0,1]$.

A set $K$ is said to be $p$-starshaped (see [6]) where $p \in K$, provided $W(x, p, \lambda) \in K$ for all $x \in K$ and $\lambda \in[0,1]$ i.e. the segment

$$
[p, x]=\{W(x, p, \lambda): 0 \leq \lambda \leq 1\}
$$

joining $p$ to $x$ is contained in $K$ for all $x \in K$. $K$ is said to be starshaped if it is $p$-starshaped for some $p \in K$.

Clearly, each convex set is starshaped but not conversely.

A convex metric space $(X, d)$ is said to satisfy Property (I) [6] if for all $x, y, q \in X$ and $\lambda \in$ $[0,1]$

$$
d(W(x, q, \lambda), W(y, q, \lambda)) \leq \lambda d(x, y)
$$

A normed linear space and each of its convex subsets are simple examples of convex metric spaces. There are many convex metric spaces which are not normed linear spaces (see [6], [13]). Property (I) is always satisfied in a normed linear space.

For a non-empty subset $M$ of a metric space $(X, d)$ and $x \in X$, an element $y \in M$ is said to be a best approximant of $x$ in $M$ or a best $M$-approximant to $x$ if $d(x, y)=d(x, M) \equiv$ $\inf \{d(x, y): y \in M\}$. The set of all such $y \in M$ is denoted by $P_{M}(x)$.

For a convex subset $M$ of a convex metric space $(X, d)$, a mapping $g: M \rightarrow X$ is said to be affine if for all $x, y \in M, g(W(x, y, \lambda))=W(g x, g y, \lambda)$ for all $\lambda \in[0,1] . g$ is said to be affine with respect to $p \in M$ if $g(W(x, p, \lambda))=W(g x, g p, \lambda)$ for all $x \in M$ and $\lambda \in[0,1]$.

Suppose $M$ is a nonempty subset of a metric space $(X, d)$ and $S, T$ are self mappings of $M$. A point $x \in M$ is a common fixed (coincidence) point of $S$ and $T$ if $x=S x=T x(S x=T x)$. The set of fixed points (respectively, coincidence points) of $S$ and $T$ is denoted by $F(S, T)$ (respectively, $C(S, T)$ ). The mappings $T, S: M \rightarrow M$ are said to be

(i) commuting on $M$ if $S T x=T S x$ for all $x \in M$;

(ii) $R$-weakly commuting on $M$ if there exists a real number $R>0$ such that $d(T S x, S T x) \leq$ $R d(T x, S x)$ for all $x \in M$.

(iii) weakly compatible if they commute at their coincidence points,i.e., if $S T x=T S x$ whenever $S x=T x$;

(iv) asymptotically $S$-nonexpansive if there exists a sequence $\left\{k_{n}\right\}$ of real numbers in $[1, \infty)$ with $k_{n} \geq k_{n+1}, k_{n} \rightarrow 1$ as $n \rightarrow \infty$ such that $d\left(T^{n}(x), T^{n}(y)\right) \leq k_{n} d(S x, S y)$, for all $x, y \in M$. 
If $S=$ identity mapping in (iv), then $T$ is said to be an asymptotically nonexpansive mapping and further, if $k_{n}=1$ for all $n \in \mathbb{N}$, then $T$ is known as nonexpansive.

$T$ is said to be uniformly asymptotically regular on $M$ if, for each $\epsilon>0$, there exists a positive integer $N$ such that $d\left(T^{n}(x), T^{n}(y)\right)<\epsilon$ for all $n \geq N$ and for all $x, y \in M$.

Suppose $(X, d)$ is a convex metric space, $M$ a $q$-starshaped subset with $q \in F(S) \cap M$ and is both $T$ - and $S$-invariant. Then $T$ and $S$ are called

(i) $R$-subweakly commuting (see [11]) on $M$ if for all $x \in M$, there exists a real number $R>0$ such that $d(T S x, S T x) \leq R \operatorname{dist}(S x, W(T x, q, k)), k \in[0,1]$;

(ii) $C_{q}$-commuting [1] if $S T x=T S x$ for all $x \in C_{q}(S, T)$, where $C_{q}(S, T)=\cup\left\{C\left(S, T_{k}\right): 0 \leq k \leq\right.$ $1\}$ and $T_{k} x=\{W(T x, q, k): 0 \leq k \leq 1\}$.

(iii) generalized asymptotically $S$-nonexpansive (see [12]) if there exists a sequence $\left\{k_{n}\right\}$ of real numbers in $[1, \infty)$ with $k_{n} \geq k_{n+1}, k_{n} \rightarrow 1$ as $n \rightarrow \infty$ such that

$$
\begin{aligned}
d\left(T^{n} x, T^{n} y\right) \leq & k_{n} \max \left\{d(S x, S y), \operatorname{dist}\left(S x,\left[T^{n} x, q\right]\right), \operatorname{dist}\left(S y,\left[T^{n} y, q\right]\right),\right. \\
& \left.\frac{1}{2}\left[\operatorname{dist}\left(S x,\left[T^{n} y, q\right]\right)+\operatorname{dist}\left(S y,\left[T^{n} x, q\right]\right)\right]\right\}
\end{aligned}
$$

for all $x, y \in M$.

Clearly, $C_{q}$-commuting maps are weakly compatible. However, converse is not true.

Example 2.1 ([1]). Let $X=\mathbb{R}$ be endowed with the usual metric and $M=[0, \infty)$. Define $T, S$ : $M \rightarrow M$ by $T x=x^{2}$ for all $x \neq 2$ and $T 2=1$; and $S x=2 x$ for all $x \in M$. Then $M$ is $q$-starshaped with $q=0, C(T, S)=\{0\}$ and $C_{q}(T, S)=\{0\} \cup[2, \infty)$. Moreover, $T$ and $S$ are weakly compatible but not $C_{q}$-commuting.

Clearly, $R$-subweakly commuting mappings are $C_{q}$-commuting but converse does not hold.

Example 2.2 ([1]). Let $X=\mathbb{R}$ be endowed with the usual metric and $M=[0, \infty)$. Define $T, S$ : $M \rightarrow M$ by $T x=\frac{1}{2}$ if $0 \leq x<1$ and $T x=x^{2}$ if $x \geq 1$; and $S x=\frac{x}{2}$ if $0 \leq x<1$ and $S x=x$ if $x \geq 1$. Then $M$ is $q$-starshaped with $q=1$, and $C_{q}(T, S)=[1, \infty)$. Moreover $S$ and $T$ are $C_{q^{-}}$ commuting but not $R$-subweakly commuting.

If $T$ and $S$ are $C_{q}$-commuting on $M$, then $S T^{n} x=T^{n} S x$ for all $x \in C_{q}\left(S, T^{n}\right)$, where $C_{q}\left(S, T^{n}\right)=\cup\left\{C\left(S, T_{k_{n}}\right): 0 \leq k_{n} \leq 1\right\}$ and $T_{k_{n}} x=\left\{W\left(T x, q, k_{n}\right): 0 \leq k_{n} \leq 1\right\}$.

Example 2.3 ([12]). Let $X=\mathbb{R}$ be endowed with the usual metric and $M=[1, \infty)$. Then $M$ is $q$ starshaped with $q=1$. Define $T, S: M \rightarrow M$ by $T x=2 x^{2}-1$ and $S x=4 x-3$ for all $x \in M$. Then $C_{q}(T, S)=[1, \infty)$ and the pair is $C_{q}$-commuting on $M$. Now $C_{q}\left(T, S^{n}\right)=[1, \infty)$ for all $n \geq 1$. It is easy to verify that $T S^{n} x=S^{n} T x$ for all $x \in c_{q}\left(T, S^{n}\right)$ and for each $n$. 


\section{Main Results}

To prove the main results, we need the following lemma. For normed linear spaces this lemma was proved in [12] and the proof can be easily extended to metric spaces.

Lemma 3.1. Let $M$ be a nonempty closed subset of a metric space $(X, d)$. Let $f, T: M \rightarrow M$ be self mappings, $q \in F(f)$ and $T(M \backslash\{q\}) \subset f(M) \backslash\{q\}$. Suppose there exists $k \in(0,1)$ such that

$$
d(T x, T y) \leq k \max \left\{d(f x, f y), d(f x, T x), d(f y, T y), \frac{1}{2}(d(f x, T y)+d(f y, T x))\right\}
$$

for all $x, y \in M$. Further, if $T$ is continuous, $c l[T(M \backslash\{q\})]$ is complete, $f$ and $T$ are weakly compatible on $M \backslash\{q\}$, then $F(f) \cap F(T)$ is singleton.

The following theorem extends and generalizes the corresponding results of Al-Thagafi and Shahzad ([1]-Theorem 2.2), Hussain and Rhoades ([7]-Theorem 2.2) and of Vijayaraju and Hemavathy ([12]-Theorem 3.1).

Theorem 3.2. Let $M$ be a nonempty subset of a convex metric space $(X, d)$ with Property (I) and $T$ and $f$ are self mappings of $M$. Suppose that $M$ is $q$-starshaped with $q \in F(f)$ and $f$ is continuous and affine with respect to $q$. If $T$ and $f$ are $C_{q}$-commuting on $M \backslash\{q\}, c l[T(M \backslash\{q\})]$ is compact, $c l[T(M)] \subseteq f(M) \backslash\{q\}$, and $T$ is continuous, uniformly asymptotically regular and generalized asymptotically $f$-nonexpansive i.e. it satisfies,

$$
\begin{aligned}
d\left(T^{n} x, T^{n} y\right) \leq & \mu_{n} \max \left\{d(f x, f y), \operatorname{dist}\left(f x,\left[T^{n} x, q\right]\right), \operatorname{dist}\left(f y,\left[T^{n} y, q\right]\right),\right. \\
& \left.\frac{1}{2}\left[\operatorname{dist}\left(f x,\left[T^{n} y, q\right]\right)+\operatorname{dist}\left(f y,\left[T^{n} x, q\right]\right)\right]\right\}
\end{aligned}
$$

for all $x, y \in M$, where $\mu_{n}$ is a sequence of real numbers with $\mu_{n} \geq 1$, and $\lim \mu_{n}=1$, then $F(T) \cap F(f) \neq \varnothing$.

Proof. Let $\left(\lambda_{n}\right)$ be a sequence of real numbers such that $0 \leq \lambda_{n}<1$ and $\lambda_{n} \rightarrow 1$. Take $k_{n}=\frac{\lambda_{n}}{\mu_{n}}$, then $\left(k_{n}\right)$ is a sequence of real numbers such that $0 \leq k_{n}<1$, and $k_{n} \rightarrow 1$. Define $T_{n}$ as $T_{n} x=W\left[T^{n} x, q, k_{n}\right]$ for all $x \in M$ and for each $n \geq 1$. As $M$ is $q$-starshaped, $f$ is affine with respect to $q$ and $c l[T(M \backslash\{q\})] \subseteq f(M) \backslash\{q\}, T_{n}$ is a self mapping of $M$ and $c l\left[T_{n}(M \backslash\{q\})\right] \subseteq f(M) \backslash\{q\}$ for each $n$. Since $T$ and $f$ are $C_{q}$-commuting, $f T^{n} x=T^{n} f x$ for all $x \in C_{q}\left(f, T^{n}\right)$. As $f$ is affine with respect to $q$, it follows that for each $x \in C_{q}(f, T), f T_{n} x=$ $f\left(W\left[T^{n} x, q, k_{n}\right]\right)=W\left[f T^{n} x, f q, k_{n}\right]=W\left[T^{n} f x, f q, k_{n}\right]=T_{n} f x$. Thus $f T_{n} x=T_{n} f x$ for each $x \in C\left(f, T_{n}\right) \subseteq C_{q}\left(f, T^{n}\right)$. Hence the pair $f$ and $T_{n}$ are weakly compatible for all $n$. Further, we have

$$
d\left(T_{n} x, T_{n} y\right)=d\left(W\left[T^{n} x, q, k_{n}\right], W\left[T^{n} y, q, k_{n}\right]\right)
$$




$$
\begin{aligned}
\leq & k_{n} d\left(T^{n} x, T^{n} y\right) \\
\leq & k_{n} \mu_{n} \max \left\{d(f x, f y), \operatorname{dist}\left(f x,\left[T^{n} x, q\right]\right), \operatorname{dist}\left(f y,\left[T^{n} y, q\right]\right),\right. \\
& \left.\frac{1}{2}\left[\operatorname{dist}\left(f x,\left[T^{n} y, q\right]\right)+\operatorname{dist}\left(f y,\left[T^{n} x, q\right]\right)\right]\right\} \\
\leq & \lambda_{n} \max \left\{d(f x, f y), d\left(f x, T_{n} x\right), d\left(f y, T_{n} y\right), \frac{1}{2}\left[d\left(f x, T_{n} y\right)+d\left(f y, T_{n} x\right)\right]\right\}
\end{aligned}
$$

for all $x, y \in M$.

As $\operatorname{cl}[T(M \backslash\{q\})]$ is compact, each $\operatorname{cl}\left[T_{n}(M \backslash\{q\})\right]$ is also compact. By Lemma 3.1, there exists $x_{n} \in M$ such that $f x_{n}=T_{n} x_{n}=x_{n}$. Since $\left\{T^{n} x_{n}\right\}$ is a sequence in the compact set $c l[T(M \backslash\{q\})]$, there exists a subsequence $\left\{T^{n_{i}} x_{n_{i}}\right\}$ of $\left\{T^{n} x_{n}\right\}$ such that $\left\{T^{n_{i}} x_{n_{i}}\right\} \rightarrow z$, for some $z \in c l[T(M \backslash\{q\})]$. Moreover,

$$
x_{n_{i}}=f x_{n_{i}}=T_{n_{i}} x_{n_{i}}=W\left[T^{n_{i}} x_{n_{i}}, q, k_{n_{i}}\right] \rightarrow z .
$$

Since $f$ is continuous, $x_{n_{i}}=f x_{n_{i}} \rightarrow f z$. By the uniqueness of the limit $z=f z$.

As $T$ is continuous, $T^{n_{i}} x_{n_{i}} \rightarrow T^{n_{i}} z$. Again by the uniqueness of the $\operatorname{limit}$, we have $\lim T^{n_{i}} z=$ $z$ and $\lim T^{n_{i}+1} z=T z$. Hence it follows that

$$
\begin{aligned}
d(z, T z) & \leq d\left(z, T^{n_{i}} z\right)+d\left(T^{n_{i}} z, T^{n_{i}+1} z\right)+d\left(T^{n_{i}+1} z, T z\right) \\
& \rightarrow 0 .
\end{aligned}
$$

Therefore $T z=z=f z$. Hence $F(f) \cap F(T) \neq \varnothing$.

Remark 1. When $T$ and $f$ are $R$-subweakly commuting and $T$ is $f$-nonexpansive, results similar to Theorem 3.2 were proved by Shahzad [11] for normed linear spaces and by the authors in [9] for convex metric spaces.

The following corollary extends Theorem 2.2 of Al-Thagafi and Shahzad [1] to asymptotically nonexpansive mappings. For normed linear spaces, the following result is given in [12].

Corollary 3.3. Let $T$ and $f$ be self mappings of a nonempty subset $M$ of a convex metric space $(X, d)$ with Property (I). Suppose that $M$ is $q$-starshaped with $q \in F(f), f$ is continuous and affine with respect to $q, c l[T(M \backslash\{q\})]$ is compact and $c l[T(M)] \subseteq f(M) \backslash\{q\}$. If $T$ and $f$ are $C_{q}$-commuting on $M \backslash\{q\}$ and $T$ is uniformly asymptotically regular and asymptotically nonexpansive with sequence $\left\{\mu_{n}\right\}$, where $\left\{\mu_{n}\right\}$ is a sequence of real numbers with $\mu_{n} \geq 1$ and $\mu_{n} \rightarrow 1$, then $F(T) \cap F(f) \neq \varnothing$.

We shall now give an application of Theorem 3.2 to the set of best approximation. For this, we need the following result. 
Proposition 3.4. If $M$ is a subset of a convex metric space $(X, d), u \in X \backslash M$ and $y \in P_{M}(u)$, then the line segment $\{W(y, u, \lambda): 0<\lambda<1\}$ and the set $M$ are disjoint.

Proof. Since $y \in P_{M}(u)$, consider

$$
\begin{aligned}
d(u, W(y, u, \lambda)) & \leq \lambda d(u, y) \\
& <d(u, M), \text { for every } 0<\lambda<1
\end{aligned}
$$

This implies that $W(y, u, \lambda) \notin M$ for any $\lambda, 0<\lambda<1$. Therefore the line segment $\{W(y, u, \lambda)$ : $0<\lambda<1\}$ and the set $M$ are disjoint.

Taking $G=M \backslash\{q\}$ for some $q \in M$, we have the following result.

Theorem 3.5. Let $M$ be a nonempty subset of a convex metric space $(X, d)$ with Property (I) and $T$ and $f$ are self mappings of $X$ such that $T(\partial G \cap G) \subseteq G$ and $u \in F(T) \cap F(f)$ for some $u \in X \backslash M$, where $\partial G$ denotes boundary of $G$. Suppose that $P_{G}(u)$ is closed and $q$-starshaped and $f$ is affine with respect to $q \in F(f)$ with $f\left(P_{G}(u)\right)=P_{G}(u)$. If $T$ and $f$ are continuous, $C_{q}$-commuting on $P_{G}(u) \cup\{u\}$ satisfying $d(T x, T u) \leq d(f x, f u), c l T\left(P_{G}(u)\right.$ is compact, and $T$ is uniformly asymptotically regular and generalized asymptotically $f$-nonexpansive mapping for all $x, y \in P_{G}(u)$, then $P_{G}(u) \cap F(T) \cap F(f) \neq \varnothing$.

Proof. Let $x \in D=P_{G}(u)$, then for any $k \in(0,1]$, we have

$$
d(W(u, x, k), u) \leq k d(u, u)+(1-k) d(x, u)=(1-k) d(x, u)<\operatorname{dist}(u, G) .
$$

It follows from Proposition 3.4 that the open line segment $\{W(u, x, \lambda): 0<\lambda<1\}$ and the set $G$ are disjoint. Thus $x$ is not in the interior of $G$ and so $x \in \partial G \cap G$. Since $T(\partial G \cap G) \subset G, T x$ must be in $G$. Also, $f\left(P_{G}(u)\right)=P_{G}(u), f x \in P_{G}(u), u \in F(T) \cap F(f)$, we have

$$
d(T x, u)=d(T x, T u) \leq d(f x, f u)=d(f x, u) \leq \operatorname{dist}(u, G) .
$$

This implies that $T x \in P_{G}(u)$. Consequently, $D=P_{G}(u)$ is $T$ invariant. Hence by Theorem 3.2, there exists $z \in P_{G}(u)$ such that $P_{G}(u) \cap F(T) \cap F(f) \neq \varnothing$.

Remark 2. Theorem 3.5 extends the corresponding results of [8], [9], [10] and [11] to generalized asymptotically $f$-nonexpansive mappings.

\section{Acknowledgements}

The authors are thankful to the learned referee for careful reading and very valuable suggestions leading to an improvement of the paper. 


\section{References}

[1] M. A. Al-Thagafi and N. Shahzad, Noncommuting selfmappings and invariant approximations, Nonlinear Analysis, 64(2006), 2778-2786.

[2] I. Beg, D. R. Sahu and S. D. Diwan, Approximation of fixed points of uniformly R-subweakly commuting mappings, J. Math, Anal. Appl., 324(2006), 1105-1114.

[3] S. Chandok and T. D. Narang, Common fixed points and invariant approximation for Gregus type contraction mappings, Rendiconti Circolo Mat. Palermo, 60(2011), 203-214.

[4] S. Chandok, Some common fixed point theorems for Ciric type contractive mappings, Tamkang J. Math., 43(2012), No.2, 187-202.

[5] K. Goebel and W. A. Kirk, A fixed point theorem for asymptotically nonexpansive mappings, Proc. Amer. Math. Soc., 35(1972), 171-174.

[6] M. D. Guay, K. L. Singh and J. H. M. Whitfield, Fixed point theorems for nonexpansive mappings in convex metric spaces, Proc. Conference on nonlinear analysis (Ed. S.P.Singh and J. H. Bury) Marcel Dekker 80(1982), 179-189.

[7] N. Hussain and B. E. Rhoades, $C_{q}$-commuting mappings and invariant approximations, Fixed Point Theory Appl., 2006(2006), Article ID 24543, 1-9.

[8] T. D. Narang and S. Chandok, Fixed points of quasi-nonexpansive mappings and best approximation, Selcuk J. Appl. Math., 10(2009), 77-82.

[9] T. D. Narang and S. Chandok, Common fixed points and invariant approximation of R-subweakly commuting maps in convex metric spaces, Ukrainian Math. J., 62 (2010), 1367-1376/1585-1596.

[10] T. D. Narang and S. Chandok, Common fixed points and invariant approximation of pointwise R-subweakly commuting maps on nonconvex sets, General Math., 4(2010), 109-125.

[11] N. Shahzad, Invariant approximations and R-subweakly commuting maps, J. Math. Anal. Appl., 257(2001), 39-45.

[12] P. Vijayaraju and R. Hemavathy, Common fixed point theorem for noncommuting mapping satisfying a generalized asymptotically nonexpansive condition, Anal. Theory Appl., 24(2008), 211-224.

[13] W. Takahashi, A convexity in metric space and nonexpansive mappings I, Kodai Math. Sem. Rep., 22(1970), 142-149.

Department of Applied Sciences, Khalsa College of Engineering \& Technology (Punjab Technical University), Ranjit Avenue, Amritsar-143001, India.

E-mail: chansok.s@gmail.com

Department of Mathematics, Guru Nanak Dev University, Amritsar-143005, India.

E-mail: tdnarang1948@yahoo.co.in 